Int. J. Environ. Res. Public Health 2006, 3(3), 286-291

International Journal of

Environmental Research and Public Health

ISSN 1661-7827

www.ijerph.org

(c) 2006 by MDPI

\title{
Free Radicals: Emerging Challenge in Environmental Health Research in Childhood and Neonatal Disorders
}

\author{
B. Sharda* \\ Dept of Pediatrics, RNT Medical College, Krishna, 5 Charak Marg, Nr. Charak Hostel, Udaipur, India. \\ *Correspondence to Dr. B. Sharda. E-mail: shardabdoc@hotmail.com,shardabdoc@yahoo.com
}

Received: 10 October 2005 / Accepted: 06 July 2006 / Published: 30 September 2006

\begin{abstract}
Infants and children may undergo severe oxidative stress due to disease state, pre-existing nutritional status, frequent use of oxygen, and lower levels of antioxidant defenses. Antioxidant defenses, made up of intracellular and extra-cellular components, work synergistically to prevent oxidative damage. Total antioxidant activity (TAA) was analyzed by method of ferric reducing antioxidant power assay (FRAP). Patients admitted in Pediatric Dept, RNT Medical College, Udaipur, India were selected for these studies. TAA level in neonates with hypoxic-ischemic-encephalopathy (HIE) stage III and in poor outcome cases was significantly low. Erythrocyte SOD activity level was low in pre-term neonates. TAA level in severely malnourished children at the time of hospital admission was low. This low antioxidant level in severely malnourished children could be multi-factorial viz. low zinc, selenium, vitamin A \& C deficiency, recurrent infections, elevated free iron and chronic starvation stage. Delayed recovery of oxidant injury may lead to delayed incomplete recovery at cellular level. In a study of 29 tuberculosis patients TAA level was found to be low in tubercular patients compared with control. TAA level decreased more in CNS tuberculosis compared with other system tuberculosis. In a study of nutritional tremor syndrome TAA, ascorbic acid and $\alpha$-tocopherol levels were low during pre-tremor phase compared with tremor phase (ATS). Pre-term neonates have incompletely developed antioxidant defenses and are deficient in vitamin E, which is normally derived from maternal circulation at the end of $3^{\text {rd }}$ trimester. Therefore, decreased TAA level in HIE with poor outcome indicates addition of antioxidants in therapeutic strategy. Since rise in TAA in antioxidant supplemented group of severely malnutrition children was higher with good outcome compared with nonsupplemented group it would be prudent to supplement antioxidant during nutritional management. These studies have shown that health benefits can be obtained by children with a reduced risk of disease from supplements of antioxidant nutrients. The amounts of optimal supplements in these disorders, whether pharmacologic or large, are to be determined. Further work is needed to show whether modest increases in nutrient intakes in children with these disorders will delay or prevent the complications and improve the outcome. Therefore, available evidence regarding health benefits to be achieved by supplementing antioxidant nutrients is encouraging. Free radical injury and antioxidant deficiency is more common than what we think. Severely malnourished children and children suffering from chronic infections and diseases are at several fold increased risk of antioxidant deficiency and likely to suffer from free radical injury. Appropriate interventions are required in reducing the risk associated with these observations.
\end{abstract}

Keywords: Free radicals, antioxidants, malnutrition, environmental health, hypoxia.

\section{Introduction}

Free radical formation and organ damage has been demonstrated from intrauterine hypoxia, suggesting the occurrence of significant oxidative stress starting from fetal life. As such, any unfavourable intrauterine environment resulting in growth restriction will lead to free radical generation and may cause oxidative fetal stress culminating into cellular damage starting in utero and manifesting after birth. Free radicals (FRs) are chemical species with one or more unpaired electrons in their outer orbit. FRs by damaging proteins, lipids, and DNA play a 
role in pathogenesis of diseases such as hypoxic-ischemic encephalopathy (HIE), intra-ventricular haemorrhage (IVH), retinopathy of pre-maturity (ROP), chronic lung disease (CLD), necrotizing enterocolitis (NEC), septisemia and icterus. The potential beneficial effects of antioxidants in protecting against diseases have been used as an argument for recommending increasing intakes of several nutrients above those derived by conventional methods. Antioxidants are being considered as therapeutic agents in increasingly number of childhood disorders. It is now well established that free radicals, especially superoxide $\left(\mathrm{O}_{2}{ }^{-{ }^{-}}\right)$, nitric oxide (NO), and other reactive species such as $\mathrm{H}_{2} \mathrm{O}_{2}$ are continuously produced in vivo. Free radicals are a product of tissue metabolism, and the potential damage they cause is minimized by the antioxidant capacity and repair mechanisms within the cell. Occasionally, free radical damage can occur, which if not repaired may lead to irreversible changes at cellular level [1-4].

Integrated antioxidant defenses protect tissues and are presumably in equilibrium with continuously generated ROS to maintain tissues metabolically intact most of the time. Disturbances to the system occur when production of ROS is rapidly increased, for example infection, trauma, pollutants, drugs, anesthetic agents, unusual food and oxygen administration. The degree of damage resulting from temporary imbalance depends on the ability of antioxidant systems to respond to the oxidant or prooxidant load. Fruits and vegetables are good sources of many antioxidants. Hence, it is believed that a healthful diet maintains the exogenous antioxidants at or near optimal levels, thus reducing the risk of tissue damage. The most prominent representatives of dietary antioxidants are vitamin $\mathrm{C}$, tocopherols, carotenoids, and flavonoids. The components of biological tissues are an ideal mixture of substrates for oxidation. Tissue structures, however, break down during inflammation and disease and free iron and other transition metals have been detected during these periods. Both zinc and selenium are intimately involved in protecting the body against oxidant stress. Vitamin $\mathrm{C}$ and $\mathrm{E}$ are principal nutrients which possess radical quenching properties and both are powerful antioxidants. Some groups of children have an increased risk of free radicalinitiated damage. This manuscript exposes a possible link between oxidative stress, antioxidant deficiency and neonatal and childhood disorders.

\section{Material and Methods}

Total antioxidant activity (TAA) was determined by the method of ferric reducing antioxidant power assay 1999 (FRAP) and expressed in $\mu \mathrm{mol} / \mathrm{L}$. Estimation of $\alpha-$ tocopherol was done by Baker and Frank Method 1968 and expressed in $\mathrm{mg} / \mathrm{dL}$. Ascorbic acid was estimated by Netelson method (1971) and expressed in mg/dL. Results were expressed as mean $\pm \mathrm{SD}$ and $\mathrm{p}$ value calculated. Patients attending to Dept of Pediatrics, RNT Medical College, Udaipur were selected. Diagnosis of individual disorder was based on clinical and laboratory parameters.

\section{Results and Discussion}

Neonatal \& Childhood Disorders in which Oxidative Stress Plays a Role-Current Status

Now increasing experimental and clinical data have provided sufficient evidence for the involvement of free radicals in preterm and term neonatal disorders. One of the major factors for improved survival of extremely low birth weight, preterm, and sick term neonates are due to major advances in the techniques of resuscitation and ventilation support which also require frequent use of oxygen. Although these techniques are life saving, there is now considerable evidence that they can lead to many complications partly due to free radicals and its related oxygen metabolites. Important disorders or complications included in this category are chronic lung diseases, bronchopulmonary dysplasia (BPD), retinopathy of prematurity (retrolental fibroplasia) (ROP), intraventricular hemorrhage (IVH), necrotizing enterocolitis (NEC), rhesus hemolytic disease (RHD), hypoxic/ischemic encephalopathy (HIE), septisemia, and congenital malformations.

These conditions are thought to be associated with tissue damage caused by generation of free radicals in the presence of oxygen excess and antioxidant deficiency. They may be life threatening in neonatal period or may contribute to an increased morbidity in later life. Preterm neonates have incompletely developed antioxidant defenses, and are deficient in vitamin $\mathrm{E}$, which is normally derived from the maternal circulation at the end of third trimester. It has been assumed that inflammatory response seen in oxygen therapy is associated with failure to scavenge free radicals generated in the extra-cellular fluid. A limited ability of preterm infants to cope with oxidative stress and the possible role of antioxidants in protecting against oxygen toxicity is well recognized. Free radical damage and role of antioxidants in many disorders is beyond experimental stage.

\section{Free Radicals and Hypoxic Brain Injury in Preterm and Term Neonates}

Hypoxic injury in the fetal and neonatal brain results in neonatal mortality and morbidity and long term sequel such as cerebral palsy, mental retardation, epilepsy, and learning disability. In term neonates, $1 \%$ to $4 \%$ of infants suffer birth asphyxia, and one-third manifest significant neurological deficits. Five to $10 \%$ of preterm infants weighing less than $1500 \mathrm{~g}$ exhibit major spastic motor deficits associated with perinatal damage to white matter; $25 \%$ to $50 \%$ show neuro-developmental deficits; and $25 \%$ show severe involvement of motor, cognition, and behavior functions. During hypoxia/ischemia activation of N-Methyl-D aspartate (NMDA) is associated with increased generation of oxygen free radicals. There is increased release of free radicals in the human fetus during asphyxia and changes in markers of oxidative stress after hypoxia in prenatal period [5]. A major observation is 
significantly higher plasma concentrations of free iron in hypoxic neonates with long-term sequel than neonates with normal neurological development. Plasma free iron seems to be a reliable early index of brain damage, reaching $100 \%$ sensitivity and specificity at higher concentration [6]. However, an increasing amount of data demonstrates abundant free radical release during hypoxia. Elevated free radical products in spinal fluid of VLBW infants with cerebral white matter injury have been reported [7]. A major observation is significantly higher plasma concentrations of free iron in hypoxic neonates with longterm sequel than neonates with normal neurological development. Plasma free iron seems to be a reliable early index of brain damage, reaching $100 \%$ sensitivity and specificity at higher concentration [6]. However, an increasing amount of data demonstrates abundant free radical release during hypoxia. Elevated free radical products in spinal fluid of VLBW infants with cerebral white matter injury have been reported [7].

\section{Erythrocyte SOD Activity and Birth Asphyxia}

Erythrocyte SOD activity in neonates with moderate and severe birth asphyxia was studied. Cord blood erythrocyte SOD activity was significantly high in moderate and severe birth asphyxia which declined significantly at 6 hours of age with oxygen therapy and remained low even at the end of 24 hours in severe asphyxia cases. Preterm neonates also require supplementation with zinc and copper to prevent their deficiency during subsequent growth and development when fed on breast milk [8].

Study of Antioxidants and Role of Free Radicals in Hypoxic-Ischemic Encephalopathy

Free radicals are produced during asphyxia and resuscitation process. Our study suggest that antioxidant levels are decreased in hypoxic-ischemic encephalopathy (HIE) infants during stage III (Table 1), with seizures (Table 2), and who expired or had morbidity (Table 3), presumably because of increased use by the body during oxidative stress. There was no change in the antioxidant level in asphyxia when compared with different Apgar score (Table 4). The administration of antioxidant enzymes like superoxide dismutase and catalase has been shown to reduce HIE damage and to maintain stability of blood brain barrier. These are protective only when administered many hours before the hypoxemic-ischemic insult. It is also suggested that nitric oxide is involved in the cascade of metabolic events that control the occurrence of hypoxemicischemic brain damage. Mean total antioxidant activity was significantly low in HIE cases who expired or in those who some neurological impairment at the time of discharge from hospital. If antioxidants are not provided in sufficient amounts, a relative deficiency can occur leading to further increase in free radical insult. In management of preterm neonates with birth asphyxia and hypoxic-ischemic encephalopathy antioxidant supplementation to reduce free radical damage is recommended [9-10].

Table 1: Total antioxidant level $(\mu \mathrm{mol} / \mathrm{L})$ in different stages of hypoxic ischemic encephalopathy (HIE).

\begin{tabular}{lcccc}
\hline HIE stage & $\begin{array}{c}\text { HIE } \\
n=30\end{array}$ & $\begin{array}{c}\text { Mean } \pm \text { Sontrol Mean } \pm \text { SD } \\
n=26\end{array}$ & p value \\
\hline I $\quad \mathrm{n}=9$ & $1656.55 \pm 431.33$ & $1438.61 \pm 441.65$ & $>0.05$ \\
II $\quad \mathrm{n}=14$ & $1642.57 \pm 550.15$ & $1438.61 \pm 441.65$ & $>0.05$ \\
III $\mathrm{n}=7$ & $1160.00 \pm 433.20$ & $1438.61 \pm 441.65$ & $<0.05$ \\
\hline
\end{tabular}

These observations indicate that antioxidants level is significantly low in HIE stage III.

Table 2: Total antioxidant level $(\mu \mathrm{mol} / \mathrm{L})$ in hypoxic ischemic encephalopathy (HIE) with seizures.

\begin{tabular}{lccc}
\hline $\begin{array}{l}\text { HIE } \\
\text { patients }\end{array}$ & $\begin{array}{c}\text { Seizures absent } \\
n=13\end{array}$ & $\begin{array}{c}\text { Seizures present } \\
n=17\end{array}$ & p value \\
\hline $\begin{array}{l}\text { Patients } \\
\mathrm{n}=30\end{array}$ & $1670.61 \pm 383.30$ & $1439.82 \pm 593.62$ & $<0.05$ \\
\hline
\end{tabular}

These observations indicate that antioxidants level is significantly low in HIE patients with seizures.

Table 3: Total antioxidant level $(\mu \mathrm{mol} / \mathrm{L})$ in hypoxic ischemic encephalopathy (HIE) with poor outcome.

HIE category TAAMean \pm SD $\begin{aligned} & \text { Inter-group } \\ & \text { comparison }\end{aligned}$ value

Group I.

Expired. $\mathrm{n}=7 \quad 1009.71 \pm 405.91 \quad$ Group I and II $\quad<0.01$

Group II.

Morbidity. n=11 1705.45 \pm 611.13 Group I and III $<0.05$

Group III.

Normal on $\quad 1886.75 \pm 446.68$ Group II and III $>0.05$ discharge. $\mathrm{n}=12$

These observations indicate that antioxidants level is significantly low in HIE patients with morbidity and who expired.

Table 4: Total antioxidant level $(\mu \mathrm{mol} / \mathrm{L})$ in relation to (HIE) \& Apgar score.

\begin{tabular}{lccc}
\hline $\begin{array}{l}\text { Apgar } \\
\text { score time }\end{array}$ & $\begin{array}{c}\text { Apgar score } \\
\text { range 1-3 }\end{array}$ & $\begin{array}{c}\text { Apgar score } \\
\text { range 4-6 }\end{array}$ & p value \\
\hline 1 min & $\mathrm{N}=27$ & $\mathrm{~N}=3$ & $>0.05$ \\
& $1499.70 \pm 464.05$ & $1844.33 \pm 976.97$ & \\
5 min & $\mathrm{N}=6$ & $\mathrm{~N}=22$ & $>0.05$ \\
& $1499.66 \pm 436.47$ & $1574.65 \pm 553.12$ & \\
\hline
\end{tabular}

There was no change in antioxidant level in asphyxiated neonates with variable Apgar score at 1 and 5 minutes. 
Oxidative Stress in Respiratory Distress Syndrome (RDS) and Bronchopulmonary Dysplasia (BPD)

Activation of respiratory burst is associated with the elaboration of reactive oxygen intermediates including superoxide anion and hydrogen peroxide Neonatal neutrophils have a variable in vitro efficiency in killing certain bacteria, especially neonates who are sick or immature. It has been seen that patients with chronic obstructive pulmonary disease undergo increased oxidative stress during exacerbations and in stable periods, their serum concentrations of antioxidant vitamins $\mathrm{A}$ and $\mathrm{E}$ decrease during exacerbations suggesting that administration of vitamin A and $\mathrm{E}$ may be beneficial in the prevention and treatment of its harmful effects [11]. In fact, with improvements in survival of many very-lowbirth-weight infants, the total number of infants developing BPD may actually be increasing. When rhSOD was administered intra-tracheally it is well tolerated and significantly increases SOD concentration and activity in serum, tracheal aspirates, and urine for 2 to 3 days in preterm infants with RDS. Neonates with RDS who subsequently develop chronic lung disease have lower transferrin levels than those who recover uneventfully from RDS. The fall in transferrin may be due to leakage into the alveoli caused by oxidative damage in the alveolar space. Preterm infants with lung immaturity may be like the adolescents: what the infant wants (a low $\mathrm{PCO}_{2}$ ) may not be what is good for him or his.

\section{Retrolental Fibroplasia or Retinopathy of Prematurity (ROP)}

A number of other risk factors for retinopathy of prematurity are: low birth weight, immaturity of retinal vasculature, supplemental oxygen, hypoxia, hypercapnia, indomethacin, and acidosis. It has been suggested that hyperoxic exposure leads to the formation of endothelial precursor (spindle) cells with excessively junction gaps; this in turn, effectively halts normal retinal vessel growth. On return to air breathing the remaining retinal vessels that have not been permanently occluded rapidly proliferate. These new vessels lack structural integrity and hemorrhage is common.

\section{Intraventricular Hemorrhage (IVH) and Necrotizing Enterocolitis (NEC)}

IVH is one of the most important causes of permanent and devastating disability in the sick preterm infant. Possible risk factors for IVH are: hypoxia, hypocapnia, mechanical ventilation, trauma, and i.v. sodium bicarbonate administration. Oxygen radicals have also been found to play a role in animal models with NEC. Intestinal ischemic-perfusion injury is thought to be chiefly mediated by oxygen radicals. The major sources of oxygen radicals in the intestine are: xanthine oxidase, which provides $\mathrm{H}_{2} \mathrm{O}_{2}, \mathrm{O}_{2}$ and neutrophilic NADPH oxidase, which produces ${ }^{-} \mathrm{O}_{2}$. Studies suggest that newborn bowel may be particularly vulnerable to oxygen radical injury and that the main source of these radicals may be activated neutrophils. Nitric oxide has received considerable attention to its role in NEC. The finding that L-arginine supplementation decreases the incidence of NEC in premature infants is an exciting development and holds great promise for the prevention of this disease [12].

\section{Erythrocyte SOD Activity in Hyperbilirubinemia and Septisemia}

Erythrocyte SOD activity in neonatal hyperbilirubinemia shows that there is significant reduction after 48 hours of phototherapy. It has been observed that at the time of diagnosis of septisemia erythrocyte SOD activity in TAGA and TSGA was low $(\mathrm{p}<0.01)$ while in preAGA it was significantly low $(\mathrm{p}<0.001)$ even after 3 days course of antibiotics $[2,8]$.

\section{Free Radical Status in Severely Malnourished Children}

In developing countries an estimated 50.6 million children under the age of 5 years are malnourished, and those who are severely malnourished and admitted to hospitals face $30-50 \%$ mortality. Why some children develop edematous PEM and others develop nonedematous PEM is unknown. Free radical has been proposed as an important factor in the development of clinical kwashiorkor or edematous PEM. Low serum zinc in malnutrition has been reported earlier [13].

Table 5: Total antioxidant activity level $(\mu \mathrm{mol} / \mathrm{L})$ in control, marasmus \& kwashiorkor on presentation, $1^{\text {st }} \&$ $2^{\text {nd }}$ follow up.

\begin{tabular}{|c|c|c|c|}
\hline \multirow{2}{*}{$\begin{array}{l}\text { Subjects } \\
\text { Control } n=16\end{array}$} & \multirow{2}{*}{ Malnutrition } & \multirow{2}{*}{$\frac{M e a n+S D}{591.88 \pm 172.59}$} & \multirow{2}{*}{$P$ value } \\
\hline & & & \\
\hline \multirow{3}{*}{$\begin{array}{l}\text { On } \\
\text { presentation }\end{array}$} & $\begin{array}{l}\text { Marasmus \& } \\
\text { Kwashiorkor } n=35\end{array}$ & $322.93 \pm 114.91$ & $<0.001$ \\
\hline & Marasmus n=22 & $374.18 \pm 103.43$ & $<0.001$ \\
\hline & Kwashiorkor $n=13$ & $236.20 \pm 75.55$ & $<0.001$ \\
\hline \multirow{3}{*}{$1^{\text {st }}$ follow up } & $\begin{array}{l}\text { Marasmus \& } \\
\text { Kwashiorkor } \mathrm{n}=18\end{array}$ & $385.55 \pm 91.68$ & $<0.001$ \\
\hline & Marasmus n=9 & $397.33 \pm 95.12$ & $<0.001$ \\
\hline & Kwashiorkor $n=9$ & $373.75 \pm 92.18$ & $<0.001$ \\
\hline \multirow{3}{*}{$2^{\text {nd }}$ follow up } & $\begin{array}{l}\text { Marasmus \& } \\
\text { Kwashiorkor } n=10\end{array}$ & $450.33 \pm 85.77$ & $<0.001$ \\
\hline & Marasmus n=5 & $430.30 \pm 78.43$ & $<0.05$ \\
\hline & Kwashiorkor $\mathrm{n}=5$ & $436.36 \pm 101.86$ & $<0.05$ \\
\hline
\end{tabular}

Antioxidant level was significantly low in marasmus and kwashiorkor patients on presentation and during $1^{\text {st }}$ and $2^{\text {nd }}$ follow up. 
Table 5 shows decreased TAA level in severely malnourished children compared with control. Decreased TAA level in these malnourished children seems to be multi factorial like low serum zinc, vitamin A, ascorbic acid, selenium, recurrent infections, elevated free iron, and chronic starvation stage. Low antioxidant status in PEM has been reported by others also [14]. Rise in TAA level in antioxidant supplemented was significantly higher $(\mathrm{p}<0.05)$ and even at the end of 2 months did not return to control level. Table 6 shows the TAA level in marasmus and kwashiorkor. Rise in TAA level was significantly higher in the group with therapeutic antioxidant during the $1^{\text {st }}$ and $2^{\text {nd }}$ follow-ups.

Table 6: Total antioxidant activity level $(\mu \mathrm{mol} / \mathrm{L})$ in marasmus and kwashiorkor with and without antioxidant supplementation during follow up.

\begin{tabular}{|c|c|c|}
\hline Subjects & $M e a n \pm S D$ & $P$ value \\
\hline $\begin{array}{l}1^{\text {st }} \text { follow up with } \\
\text { supplementation } n=13\end{array}$ & $405.61 \pm 95.47$ & $<0.05$ \\
\hline $\begin{array}{l}1^{\text {st }} \text { follow up without } \\
\text { supplementation } n=5\end{array}$ & $373.39 \pm 69.57$ & NS \\
\hline $\begin{array}{l}\text { 2nd follow up with } \\
\text { supplementation } n=8\end{array}$ & $428.03 \pm 83.28$ & $<0.05$ \\
\hline $\begin{array}{l}2^{\text {nd }} \text { follow up without } \\
\text { supplementation } n=2\end{array}$ & $454.55 \pm 128.6$ & NS \\
\hline On presentation $n=35$ & $322.93 \pm 114.91$ & \\
\hline
\end{tabular}

Rise in antioxidant level was significantly higher in therapeutic antioxidant supplemented group during $1^{\text {st }}$ and $2^{\text {nd }}$ follow up.

\section{Role of Free Radicals in Childhood Tuberculosis}

In our study of 29 tuberculosis cases (18 of respiratory system, 6 of lymph nodes, and 5 of central nervous system) there was decreased TAA observed which was statistically significant. Serum ascorbic acid and $\alpha$-tocopherol levels were also significantly low in these tuberculosis cases compared with healthy controls (Table 7). It was also observed that TAA, ascorbic acid, and $\alpha$-tocopherol were found to be more decreased in central nervous tuberculosis compared with respiratory system and lymph node tuberculosis.

Table 7: Total antioxidant, ascorbic acid, and $\alpha$-tocopherol levels in tuberculosis.

\begin{tabular}{lcccc}
\hline & $\begin{array}{c}\text { Tuberculosis } \\
\mathrm{n}=29\end{array}$ & Control $\mathrm{n}=20$ & $\mathrm{p}$ value \\
\hline TAA & $983.55 \pm 218.35$ & $1402.50 \pm 512.18$ & $<0.001$ \\
$\mu \mathrm{mol} / \mathrm{L}$ & & & \\
$\begin{array}{l}\alpha \text {-tocopherol } \\
\mathrm{mg} / \mathrm{dL}\end{array}$ & $0.91 \pm 0.51$ & $1.30 \pm 0.55$ & $<0.001$ \\
$\begin{array}{l}\text { Ascorbic } \\
\text { acid mg/dL }\end{array}$ & $0.27 \pm 0.17$ & $0.43 \pm 0.14$ & $<0.001$ \\
\hline
\end{tabular}

These data indicate significant lower values of antioxidant, ascorbic acid and tocopherol in tuberculosis.

\section{Anemia Tremor Syndrome and Free Radicals}

Anemia tremor syndrome (ATS) is a peculiar disorder seen in Indian children who are exclusively breast fed even after one year of age and characterized by hyperpigmentation, hair discoloration, and regression of mile stones. Consistency of these clinical features in specified cast and season makes it a distinct clinical disorder in this area. Since oxidative damage may play a role in neurodegenerative diseases which cause cognitive impairment, including dementia, stroke, and regression of mile stones in children hence, oxidative stress was assessed in these children. Ascorbic acid and $\alpha$-tocopherol levels were significantly low in ATS patients compared with control. TAA during pre-tremor phase was significantly low in these patients (Table 8).

Table 8: Total antioxidant, ascorbic acid, and $\alpha$-tocopherol level in anemia tremor syndrome.

\begin{tabular}{lccc}
\hline & ATS $n=22$ & $\begin{array}{c}\text { Pre-tremor } \\
n=16\end{array}$ & P value \\
\hline $\begin{array}{l}\text { TAA } \\
\mu \mathrm{mol} / \mathrm{L}\end{array}$ & $644.23 \pm 226.84$ & $501.88 \pm 172.59$ & $<0.01$ \\
$\begin{array}{l}\text { Ascorbic } \\
\text { acid mg/dL }\end{array}$ & $0.38 \pm 0.21$ & $0.58 \pm 0.29$ & $<0.05$ \\
$\begin{array}{l}\alpha \text {-tocopherol } \\
\text { mg/dl }\end{array}$ & $0.70 \pm 0.26$ & $2.02 \pm 0.84$ & $<0.001$ \\
\hline A &
\end{tabular}

Antioxidant, ascorbic acid and $\alpha$-tocopherol levels were significantly low in patients of anemia tremor syndrome.

Acknowledgement: I am thankful for the assistance provided by Prof. P. P. Singh, Dr Vishal Gupta, Dr Tanu Revri, and Dr Vinay Vyas.

\section{References}

1. Sharda, B.: Free Radicals: Pediatrician's Perspective. In: Recent Advances in Pediatrics. Gupte, S. (ed.). Jaypee Brothers: New Delhi, 1999; Vol. 9, pp. 217-236.

2. Sharda, B.: Free Radicals \& Neonatal Disorders. In: Recent Advances in Pediatrics. Gupte, S. (ed.). Jaypee Brothers: New Delhi, 2002; Vol. 12, pp. 206-221.

3. Sharda, B.: Free Radicals: Newer Aspects. In: Toxicology and Environmental Health. Vohora \& Agarwal. (eds.). Asiatech Publ. Inc.: New Delhi, 1999: pp. 97-114.

4. Sharda, B.: Nitric Oxide. In: Recent Advances in Pediatrics. Gupte, S. (ed.). Jaype Brothers: New Delhi, 2000; Vol. 10, pp. 207-220.

5. Saliba, E.; Henrot, A.: Inflammatory mediators and neonatal brain damage. Biol. Neonate 2001, 79, 224227.

6. Ozawa, H.; Nishida, A.; Mito, T.; Takashima, S.: Development of ferritin-positive cells in cerebrum of human brain. Pediatr. Neurol. 1994, 10, 44-88.

7. Inder, T.; Mocatta, T.; Darlow, B.; Spencer, C.; Volpe, J. J.; Winterbourn, C.: Elevated free radical products in 
cerebrospinal fluid of VLBW infants with cerebral white matter injury. Pediatr. Resear. 2002, 52, 213-218.

8. Sharda, B.; Adhikari, R.: Erythrocyte SOD activity assay and zinc and copper levels in preterm neonates. XXII International Congress of Pediatrics, Amsterdam, August 9-14, 1998, pp. 142.

9. Sharda, B.: Hypoxic-Ischemic Encephalopathy. In: Recent Advances in Pediatrics. Gupte, S. (Ed.). Jaypee Brothers: New Delhi, 2002; Vol. 12, pp. 120-131.

10. Avery, G. B.; Fletcher, M. A.; Macdonals, M. G.: Neonatology: Pathophysiology and Management of the Newborn. In: Hill, A.; Volpe, J. J. (Eds.). Lippincott Williams and Wilkinson: London, 1999; pp. 1231-1252.
11. Tug, T.; Karatas, F.; Terzi, S. M.: Antioxidant vitamins in acute exacerbation and stable periods of patients with chronic obstructive pulmonary disease. Clinical Investig. Med., 2004, 27, 123-128.

12. Amin, H. J.; Zamora, S. A.; McMillan, D. D.; Fick, G. H.; Butzner, J. D.; Parsons. H. G.; et al.: Arginine supplementation prevents necrotizing enterocolitis in the premature infant. J. Pediatr., 2002, 140, 425-431.

13. Sharda, B.; Bhandari, B.: Serum zinc in protein calorie malnutrition. Indian Pediatr., 1977, 14,195-196.

14. Ashour, M. N.; Salem, S. I.; El-Gadban, H. M.; Flwan, N. M.; Basu, T. K.: Antioxidant status in children with protein-energy malnutrition (PEM) in Cairo, Egypt. Euro. J. Clin. Nutr. 1999, 52, 669-673. 